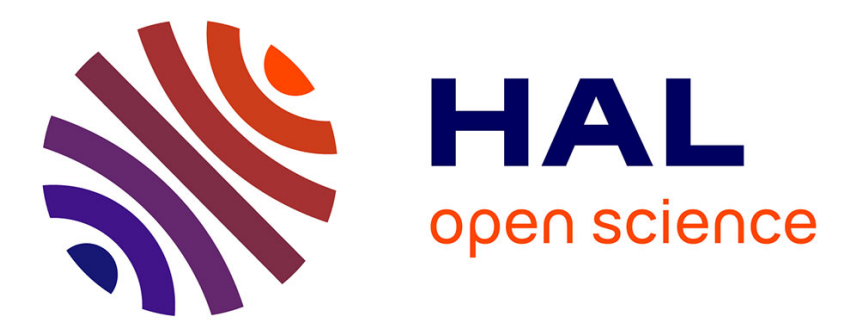

\title{
Optical Properties of Guanine Nanowires: Experimental and Theoretical Study
}

P. Changenet-Barret, E. Emanuele, T. Gustavsson, R. Improta, A. B. Kotlyar, D. Markovitsi, I. Vaya, K. Zakrzewska, D. Zikich

\section{- To cite this version:}

P. Changenet-Barret, E. Emanuele, T. Gustavsson, R. Improta, A. B. Kotlyar, et al.. Optical Properties of Guanine Nanowires: Experimental and Theoretical Study. Journal of Physical Chemistry C, 2010, 114, pp.14339-14346. 10.1021/jp102106d . hal-00516388

\section{HAL Id: hal-00516388 \\ https://hal.science/hal-00516388}

Submitted on 10 Sep 2010

HAL is a multi-disciplinary open access archive for the deposit and dissemination of scientific research documents, whether they are published or not. The documents may come from teaching and research institutions in France or abroad, or from public or private research centers.
L'archive ouverte pluridisciplinaire HAL, est destinée au dépôt et à la diffusion de documents scientifiques de niveau recherche, publiés ou non, émanant des établissements d'enseignement et de recherche français ou étrangers, des laboratoires publics ou privés. 


\section{Optical properties of guanine nanowires: experimental and theoretical study}

Pascale Changenet-Barret, ${ }^{1}$ Emanuela Emanuele, ${ }^{1}$ Thomas Gustavsson, ${ }^{1}$ Roberto Improta, ${ }^{1 \dagger}$ Alexander B. Kotlyar, ${ }^{2}$ Dimitra Markovitsi, ${ }^{* 1}$ Ignacio Vaya, ${ }^{1}$ Krystyna Zakrzewska ${ }^{3}$ and Dragoslav Zikich ${ }^{2}$

Laboratoire Francis Perrin, CEA/DSM/IRAMIS/SPAM - CNRS URA 2453, 91191 Gif-surYvette, France

Department of Biochemistry, Georges S. Wise Faculty of Life Sciences, Tel Aviv University, Ramat Aviv, 69978 Israel

Institut de Biologie et Chimie des Protéines, UMR5086, 69367 Lyon, France

\footnotetext{
${ }^{\dagger}$ Permanent address : Istituto Biostrutture e Bioimmagini-CNR Via Mezzocannone 16
} I-80134 Napoli Italy 


\begin{abstract}
Long nanowires formed by $c a .800$ guanine tetrads (G4-wires) are studied in phosphate buffer containing sodium cations. Their room temperature optical properties are compared to those of the monomeric chromophore 2-deoxyguanine monophosphate (dGMP). When going from dGMP to G4-wires, both the absorption and fluorescence spectra change. Moreover, the fluorescence quantum yield increases by a factor 7.3 whereas the average fluorescence lifetime increases by more than two orders of magnitude indicating emission associated with weakly allowed transitions. The behavior of G4-wires is interpreted in the light of a theoretical study performed in the frame of the exciton theory combining data from molecular dynamics and quantum chemistry. These calculations, carried out for a quadruplex composed of three tetrads, reveal the existence of various exciton states having different energies and oscillator strengths. The degree of delocalization of the quadruplex Franck-Condon excited states is larger than those found for longer duplexes following the same methodology. The slower excited state relaxation in G4-wires compared to dGMP is explained by emission from exciton states, possibly limited on individual tetrads, whose coherence is preserved by the reduced mobility of guanines due multiple Hoogsteen hydrogen bonds.
\end{abstract}

Keywords: G-quadruplex, guanine wires, DNA fluorescence, excitons 


\section{Introduction}

The use of DNA components as building blocks in non-conventional materials for applications in molecular electronics and optoelectronics is a rapidly emerging field. ${ }^{1,2}$ In this respect, guanines occupy a privileged position due to their ability to self-assemble into nanoscale structures. Long guanine wires (G4-wires), formed by folding a few thousand bases single poly $(\mathrm{dG})$ strand, present a high potential for use in the field of nanotechnologies. ${ }^{3,4} \mathrm{G} 4-$ wires are typical examples of G-quadruplexes in which the repetitive unit is a tetrad composed of four guanines linked together by Hoogsteen hydrogen bonds (Figure 1). Both monomeric nucleic acids and numerous guanine rich DNA sequences, including those encountered at the ends of human chromosomes (telomeres), give rise to a variety of fourstranded structures. G-quadruplexes are known to play an important role in gene expression and constitute targets for cancer therapy. ${ }^{5-9}$

Characterizing the optical properties of guanine nanowires and disentangling the various factors that may affect them is a prerequisite for the integration of such systems in optoelectronic devices. Although thousands of publications have been dedicated to structural and functional aspects related with G-quadruplexes, very little is known about their electronic excited states.

Changes in the electronic absorption spectra are currently used in a phenomenological way for monitoring the formation of G-quadruplexes. ${ }^{10}$ A qualitative interpretation of these changes was recently proposed by comparing single-stranded and four-stranded d(TGGGGT). ${ }^{11}$ The observed spectral differences were explained in terms of vertical and horizontal coupling, in analogy with $\mathrm{H}$ and $\mathrm{J}$ aggregates, respectively. But so far, this explanation was not rationalized via theoretical calculations.

The fluorescence properties of G-quadruplexes are much less explored than their absorption behavior. Three groups reported that self-association of guanine rich DNA strands 
leads to an increase of the fluorescence quantum yields. ${ }^{11-14}$ Another trend which also emerged from the time-resolved studies is that self-association of guanines results to a slowing down of the fluorescence decays. ${ }^{12} 11,13,15,16$ However, a quantitative comparison of the above mentioned observations is delicate. This difficulty is related to the systems studied as well as to the experimental conditions used by each group. For example, some data were obtained simply by mixing guanine single strands without a clear proof that quadruplex structures were indeed formed. ${ }^{12,15}$ Furthermore, the comparison between the optical properties of short and long wires is difficult because the former were stabilized by sodium ions, ${ }^{11}$ whereas the latter were either empty or filled with potassium ions. ${ }^{13}$ Metal ions are known to affect the quadruplex structure ${ }^{17}$ and, consequently, the coupling between electronic transitions of the guanine moieties. As a result, the properties of the G-quadruplex excited states and their relaxation should depend on the presence and the type of metal ions.

Here we present an experimental and theoretical study dealing with the optical properties of guanine quadruplexes in the presence of sodium cations. The choice of $\mathrm{Na}^{+}$was made in order to compare the results with those obtained previously under similar conditions both for quadruplexes ${ }^{11}$ and for DNA duplexes. ${ }^{18-22}$ The objectives of the study are to determine the absorption and fluorescence properties of long guanine wires at room temperature and to provide the first theoretical description of the electronic excited states of guanine quadruplexes. Long G4-wires are suitable models for understanding the specific behavior arising from tetrad formation and allow comparison with theoretical work. In contrast, short quadruplex structures, as for example d(TGGGGT) ${ }_{4}$ or those formed by the telomeric sequence d(TTAGGG), in addition to guanines, contain other bases which contribute to photon absorption and photon emission and blur the intrinsic properties of the tetrad core. 
The experimental work is carried out for long guanine wires composed of $c a .800$ tetrads dissolved in phosphate buffer containing $\mathrm{Na}^{+}(0.13 \mathrm{mM})$. The fluorescence decays of the G4-wires were obtained by time-correlated single photon counting (TCSPC). We stress that the fluorescence experiments were performed following specific protocols developed for DNA helices. ${ }^{23}$ This is a key point for obtaining reliable data for systems absorbing in the UV spectral domain and characterized by extremely low fluorescence quantum yields $\left(<10^{-3}\right)$.

The modeling concerns the excited states populated directly upon photon absorption (Franck-Condon states). It is carried out for short quadruplexes composed of three tetrads, d(GGG) $)_{4}$. We follow a methodology developed recently for the study of DNA duplexes ${ }^{24,25}$ in the frame of the exciton theory. ${ }^{26}$ The excited states of the quadruplex are linear combinations of the excited states associated with each monomeric guanosine chromophore composing the system. In our calculations we take into account structural disorder by considering a multitude of conformations derived from molecular dynamics simulations. We stress that such calculations, combining exciton theory with molecular dynamics simulations, cannot be performed for wires containing hundreds of tetrads.

The paper is organized as follows. First we detail the experimental and computational methods used in the study (Section 2). The experimental results are presented in Section 3, followed by the results of the calculations (Section 4). Then, we compare the experimental and calculated features of the absorption spectra and discuss the relaxation of the excited states. We finish with some concluding remarks outlining future developments.

\section{Experimental and Computational Details}

\subsection{Materials and Methods}

Long monomolecular G4-wires were prepared as previously described. ${ }^{3}$ The procedure consists of two main steps: the enzymatic synthesis and purification of continuous 2800 base dG strands, and folding of the purified G strands into G4-wires structures. The 
structures were characterized by AFM, UV spectroscopy, circular dichroism as well as by the sensitivity to enzymes. ${ }^{3,27,28}$

AFM was performed on molecules adsorbed on mica surfaces on which binding of nucleic acids is promoted by magnesium cations. Here $20 \mu \mathrm{l}$ of DNA sample in $2 \mathrm{mM}$ of Tris-acetate buffer ( $\mathrm{pH} 7.4$ ), containing $2 \mathrm{mM}$ of $\mathrm{MgCl}_{2}$, were incubated on freshly cleaved muscovite mica plates for 2 to $5 \mathrm{~min}$, washed with distilled water, and dried with nitrogen gas. AFM images were obtained with a Solver PRO atomic force microscope (NT-MDT, Russia) in a noncontact (tapping) mode using the silicon/gold-coated cantilevers (NT-MDT), $130 \mu \mathrm{m}$ long with a resonance frequency of 119 to $180 \mathrm{KHz}$. The images were "flattened"' (each line was fitted to a second-order polynomial and then subtracted from the image line) by the AFM image processing software. A typical AFM image of the synthesized G4-wires is shown in Figure 2. As seen from the image, the preparation procedure yielded G4-DNA wires, with a uniform morphology and a relatively narrow length distribution. The average contour length of the wires is approximately equal to $200 \mathrm{~nm}$. The correlation between the total amount of nucleotides in the poly $(\mathrm{dG})$-strand and the contour length of the folded structures corresponds nicely with a 4-intramolecular folding of the strand into the wire.

For the spectroscopic studies, G4-wires were dissolved in phosphate buffer (10 mM $\mathrm{NaH}_{2} \mathrm{PO}_{4}, 10 \mathrm{mM} \mathrm{Na} 2 \mathrm{HPO}_{4}, 0.1 \mathrm{M} \mathrm{NaCl} ; \mathrm{pH}$ 7.4). Ultrapure water was produced by a MILLIPORE (Milli-Q Synthesis) system. The absorbance of the samples was adjusted to 0.1 per $\mathrm{cm}$ for both steady-state and time-resolved fluorescence measurements.

Steady-state absorption and fluorescence spectra were recorded with a Perkin-Elmer Lamda 900 spectrophotometer and a SPEX Fluorolog-3 fluorimeter, respectively, using $1 \mathrm{~cm}-$ optical path quartz cells. Fluorescence spectra over the whole UV-visible spectral region were recorded in two steps: between 250 and $450 \mathrm{~nm}$ without any optical filter on the emission detection, then between 400 and $700 \mathrm{~nm}$ using a Schott GG 385 filter to eliminate 
contribution from the second order of the scattered excitation beam, Raman scattering and the UV part of the G4-wire fluorescence. Fluorescence spectra were corrected from the instrumental wavelength-dependent response after subtraction the signal arising from the pure solvent. The fluorescence quantum yield of the G4-wires was determined using thymidine-5'monophosphate $(\mathrm{TMP})$ in pure water $\left[\phi_{\mathrm{f}}=(1.54 \pm 0.11) \times 10^{-4}\right]^{29}$

Fluorescence decays were measured by TCSPC using a Becker \& Hickl GmbH PC card. The excitation source was the third harmonic $(267 \mathrm{~nm})$ of a mode-locked Ti-Sapphire laser (Coherent MIRA 900), delivering 100-fs pulses with a repetition rate of $4.75 \mathrm{MHz}$. A Schott WG 295 filter was placed in front of a SPEX monochromator. The detector was a microchannel plate (R1564 U Hamamatsu) providing an instrumental response function of 60 ps (fwhm). A Glan Thomson prism at the magic angle was placed at the excitation side. The average laser power $(0.1 \mathrm{~mW})$ was measured with a Melles Griot broadband powermeter. The irradiated area on the surface of the cell was $c a .0 .2 \mathrm{~cm}^{2}$ leading to corresponding to a pulse intensity of $2.4 \mathrm{~kW} / \mathrm{cm}^{2}$. Solutions were contained in a $10 \mathrm{~mm} \times 10 \mathrm{~mm}$ quartz cell and continuously stirred. Successive recordings with the same sample gave identical decays which were eventually merged to decrease the signal-to-noise ratio. Such a procedure allowed us to check that the measured signals were not altered during the measurements due to accumulation of photoproducts (add Figure?)

\subsection{Theoretical methodology}

The ground state conformations of 3xG4 were extracted from molecular dynamics simulations including explicitly solvent and sodium counter-ions. ${ }^{24}$ The quadruplex was constructed on the basis of the crystal structure (pdbid 352D) using four single strands d(GGG) conformation and was neutralized with $8 \mathrm{Na}^{+}$ions placed by AMBER's LEAP module of outside the tetramer and solvated with 2000 TIP3P water molecules in a truncated octahedral box. Molecular dynamics simulations were performed at constant temperature (300 
$\mathrm{K})$ and pressure ( 1 bar) using the Berendsen algorithm. ${ }^{30}$ An integration time step of 2 fs was used and all bond lengths involving hydrogens were constrained using SHAKE. ${ }^{31}$ Long-range electrostatic interactions were treated using the particle mesh Ewald (PME) approach ${ }^{32}$ with a $9 \AA$ direct space cut-off. The non-bonded pair-list was updated heuristically and the center of mass motion was removed every 10 ps during the simulation. Initially, the water molecules and ions were relaxed by energy minimization and allowed to equilibrate at $300 \mathrm{~K}$ around the fixed DNA for 100 ps at constant volume; the entire system was then heated from 100 to 300 K during 100 ps and equilibrated during 50 ps with harmonic restraints of $5.0 \mathrm{kcal} / \mathrm{mol} / \AA^{2}$ on the solute atoms at constant volume. Subsequently, the simulation was continued at constant pressure; the restraints were gradually removed over a period of $250 \mathrm{ps}$ and an unrestrained simulation followed for over $15 \mathrm{~ns}$. The coordinates were saved every 1 ps. The last nanosecond was used for the further study. 100 snapshots spaced by 10 ps were selected. In order to minimize bond length and valence angle distortions the snapshots were minimized in AMBER for 1000 cycles before being used for Poisson-Boltzmann calculations of the electrostatic energy. Non-linear solutions of the Poisson-Boltzmann equation were obtained with the DELPHI program (version 2.1). ${ }^{33}$ As in our previous calculations, ${ }^{33}$ an ionic strength of 0.145 was employed. Calculations involved 145 grid points and $80 \%$ of the box filled by the molecule, corresponding to 4.2 grid points/Å. The internal dielectric constant was taken as 2 , and the external value as 80 . The solvent accessible surface of the molecule was defined using a probe radius of $1.4 \AA$.

The excited states of the quadruplex were calculated in the frame of the exciton theory. The detailed formalism is described in reference ${ }^{34}$. We constructed the Hamiltonian matrix taking into account the two close $\pi \pi^{*}$ lying transitions of guanine $S_{0} \rightarrow S_{1}$ and $S_{0} \rightarrow$ $\mathrm{S}_{2}$. 
The excitation energy $E_{m}^{i}$ corresponding to the transition $S_{0} \rightarrow S_{i}$ of a chromophore $m$ within the quadruplex is given by:

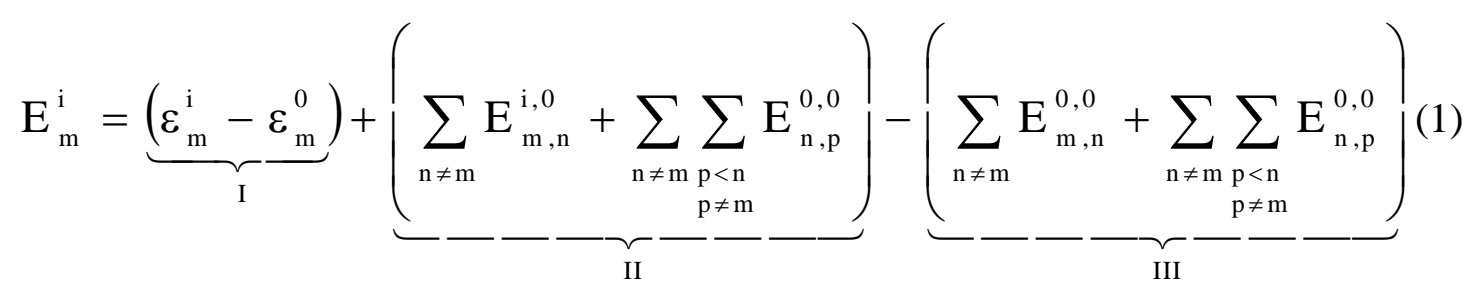

Term (I) represents the excitation energy of free monomer $m$ from its ground to its $i^{\text {th }}$ electronic state. Term (II) corresponds to the interaction energy of the system in which monomer $m$ is in its $i^{\text {th }}$ state and all others in their respective ground states. It is computed as the electrostatic energy of the system in water by solving the non-linear Poisson-Boltzmann equation with AMBER atomic charges. In this calculation, atomic charge distributions associated with the excited states of the monomers were used. Finally, term (III) is the energy of the ground-state system; it is calculated by the Poisson-Boltzmann method.

Atomic charges for the excited states were constructed from $a b$ initio calculations. We described the change in the monomers' electronic wavefunction upon excitation as a set of atomic charge differences, computed by subtraction of the CASSCF/RESP charges on the atoms of the ground state molecule from those corresponding to the excited state. The active space choose for CASSCF/RESP calculation is 14 electrons in 12 molecular orbitals. All the valence pi-orbitals and the lone pairs orbitals of the heteroatoms were included. The basis set used is cc-pVZ. The charges for the excited states of guanine were obtained by e the corresponding charge difference to the standard AMBER ground-state charges. This should account for the reorganization of the electronic system of the monomers upon excitation, while retaining the generality of the AMBER force field, which is very well suited to the study of nucleic acids. ${ }^{35}$ The calculation procedure is described in detail in the appendix of reference 24. The terms $\varepsilon_{\mathrm{m}}^{\mathrm{i}}$ were given constant values, $36000 \mathrm{~cm}^{-1}$ and $37500 \mathrm{~cm}^{-1}$, derived 
from the fits of the experimental absorption spectra of dGMP in aqueous solution with lognormal functions. ${ }^{34}$

The dipolar coupling was calculated using the atomic transition charge distribution model, according to which the off-diagonal terms are subjected to a dipolar development. ${ }^{36}$ The resulting molecular transition dipoles $\vec{\mu}=\left\langle\Psi_{\mathrm{m}}^{\mathrm{k}}|\overrightarrow{\mathrm{r}}| \Psi_{\mathrm{m}}^{0}\right\rangle$ are then decomposed onto the atomic orbitals of the molecule, in the framework of the INDO approximation. ${ }^{37}$ Atomic charges of the two transitions were derived from quantum chemistry calculations preformed on 9-methylguanine (INDO). ${ }^{34}$ They were rescaled so that the computed transition moments to match the experimental transition moments, $3.31 \mathrm{D}$ for each transition. Couplings corresponding to all guanine pairs of the quadruplex were taken into account.

Diagonalization of the exciton matrix corresponding to a given quadruplex conformation yields the eigenstates of the system which are linear combinations of the wavefunctions $\left\langle\Psi_{n}\right\rangle$ corresponding to the monomer transitions $|k\rangle=\sum_{n=1}^{N} C_{k, n}\left|\Psi_{n}\right\rangle$. Since the studied quadruplex contains 12 guanines with two transitions each, it has twenty four eigenstates $\langle\mathrm{k}>$, whose energy increases from $<1>$ to $<24>$.

\section{Experimental Results}

\subsection{Steady-sate spectra}

Figure 3 shows the normalized steady-state absorption and fluorescence spectra of G4wires together with those of dGMP. Various parameters allowing a comparison between the spectra of the wires with those of dGMP are summarized on Table 1. The spectra of Figure 3 are presented on a wavenumber scale so that to provide information about the energy of the excited states related to photon absorption and photon emission and allow comparison with the results of the calculations. In order to keep the link with the directly observed experimental data, we also give below some spectral characteristics in wavelength. 
Figure 3

The spectra in Figure 3 were obtained for the wires dissolved in $10 \mathrm{mM}$ sodium phosphate buffer $(\mathrm{pH}=7.4)$ containing $0.1 \mathrm{M} \mathrm{NaCl}$. These spectra remain practically unaltered when the $\mathrm{NaCl}$ concentration varies from 0.05 to $2.5 \mathrm{M}$ or when the $\mathrm{pH}$ ranges from 6.3 to 8.5 .

Table 1

The absorption spectrum of the wires exhibits three striking differences with respect to the spectrum of the monomeric chromophores. First, the shoulder around $36000 \mathrm{~cm}^{-1}$ (ca. 275 $\mathrm{nm}$ ) disappears and the spectral width is reduced from $7100 \mathrm{~cm}^{-1}$ to $6600 \mathrm{~cm}^{-1}$ (full width at half maximum, fwhm). Moreover, a hyperchromism appears at the red wing of the spectrum which is typical of the quadruplexes formation. ${ }^{10}$ Finally, the maximum is blue shifted from 253 to $251 \mathrm{~nm}$ corresponding to an energy difference of $315 \mathrm{~cm}^{-1}$. Interestingly, no such shift is observed for the short quadruplex $\mathrm{d}(\mathrm{TGGGGT})_{4}{ }^{11}$ In general, formation of G-quadruplexes composed by short oligomers gives rise to a decrease of the maximum molar extinction coefficient. Consequently, their melting results to an increase of the absorbance around the peak of the spectrum. As the long G4-wires studied here are prepared by biochemical methods in solution and they are extremely stable as a function of temperature, it has not been possible to determine the absolute molar absorption coefficients $(\varepsilon)$. We used $\varepsilon$ values normalized relative to the maximum in order to determine the barycenter $\mathrm{B}=\Sigma(\mathrm{E} \cdot \varepsilon) / \Sigma \varepsilon$, where $\mathrm{E}$ is the excitation energy. The B value found for the dGMP and the wires are 39100 and $39670 \mathrm{~cm}^{-1}$, respectively, revealing an overall hypsochromic shift of $570 \mathrm{~cm}^{-1}$ for the quadruplex. 
On a wavelength scale, the fluorescence spectrum of the wires recorded upon excitation at $266 \mathrm{~nm}$ peaks at $353 \mathrm{~nm}$, whereas that of dGMP is located at $334 \mathrm{~nm}$. A red shift, though smaller (6 $\mathrm{nm}$ instead of $19 \mathrm{~nm}$ ), was also reported for the d(TGGGGT) ${ }_{4}$ quadruplexes ${ }^{11}$. On a wavenumber scale (Figure 3b), the difference in the emission maxima between the monomeric chromophores and the wires is $2250 \mathrm{~cm}^{-1}$. In analogy with the absorption spectra, we determined the barycenters of the emission ones: $25500 \mathrm{~cm}^{-1}$ for the wires and $23900 \mathrm{~cm}^{-1}$ for dGMP. This means that, despite the fact that the emission maximum of the wires is located at lower energy with respect to the monomer, on average they emit photons of higher

energies. It is worth-noticing that the red tail characterizing of the guanosine emission ${ }^{38}$ is weaker in the case of the wires. For example, at $18000 \mathrm{~cm}^{-1}(\mathrm{ca} .555 \mathrm{~nm})$, the relative intensity of the spectrum compared to that at the maximum is $29 \%$ for dGMP but it dwindles down to $8 \%$ for the wires. The width (fwhm) of the wire fluorescence spectrum $\left(8300 \mathrm{~cm}^{-1}\right)$ is smaller than that of dGMP $\left(8950 \mathrm{~cm}^{-1}\right)$ by $650 \mathrm{~cm}^{-1}$. The fluorescence quantum yield of the wires is $(9.5 \pm 0.1) \times 10^{-4}$, to be compared with the value of $(1.3 \pm 0.1) \times 10^{-4}$ determined for dGMP for the same excitation wavelength $(265 \mathrm{~nm})$.

Figure 4

\subsection{Fluorescence decays}

The fluorescence decays of the wires recorded at 340 and $450 \mathrm{~nm}$ are shown in Figure 4. At both wavelengths, the signal is clearly longer than the instrumental response function given by the Raman line of water at $294 \mathrm{~nm}$. Three time constants are needed in order to fit the decays. The results of the fits with tri-exponential functions $\Sigma \mathrm{a}_{\mathrm{i}} \exp \left(-\mathrm{t} / \tau_{\mathrm{i}}\right)$ are presented on Table 1 . We also present on Table 1 the weight corresponding to each time constant $p_{j}=a_{j} \tau_{j} /$ $\left(\sum \mathrm{a}_{\mathrm{i}} \tau_{\mathrm{i}}\right)$ as well as the average lifetime $\langle\mathrm{t}\rangle=\Sigma \mathrm{a}_{\mathrm{i}} \tau_{\mathrm{i}}$. The shortest time constant $\tau_{1}$ amounts to 
31.6 and $25.8 \mathrm{ps}$ at 340 and $450 \mathrm{~nm}$, respectively. The $\tau_{2}$ values are about one order of magnitude higher (272 and 302 ps). Finally, a long component equal to $2.0 \mathrm{~ns}$ is detected at both probed wavelengths. The average lifetime is $132 \mathrm{ps}$ at $340 \mathrm{~nm}$ and $165 \mathrm{ps}$ at $450 \mathrm{~nm}$. Taking into account the relative intensities of the fluorescence spectrum at these two wavelengths, we determine a global average lifetime of 139 ps. Following the same procedure for dGMP, whose decays also vary with the emission wavelength, ${ }^{38}$ we obtain an average lifetime of 0.52 ps. By considering the fluorescence quantum yields of the two systems, we evaluate the corresponding average radiative lifetimes: $4 \mathrm{~ns}$ for dGMP, which is typical of a strong transition, and $146 \mathrm{~ns}$ for the wires, which suggests emission associated, at least partly, to weak transitions.

Table 2

\section{Theoretical Results}

\subsection{Ground state geometry}

Figure 5

Figure 5 shows top and side views corresponding to the average conformation of the $\mathrm{d}(\mathrm{GGG})_{4}$ quadruplex. The sodium cations are located in the central cavity where they can move freely. We observed that structural parameters obtained for the $\mathrm{d}(\mathrm{GGG})_{4}$ quadruplex by molecular dynamics simulations are very similar to those reported for the d(TGGGGT) 4 crystal by X ray measurements. ${ }^{39}$ For example, we determined for d(GGG) $)_{4}$ an average helical rise of $3.29 \AA$ and a helical twist of $26.4^{\circ}$ with the standard deviation (rmsd) of 0.18 and $5.72^{\circ}$, respectively. The corresponding values reported for the crystal are $3.30 \AA$ and $27.5^{\circ}$. It 
is worth-noticing that the rise corresponding to the quadruplex structure is larger and the twist is smaller compared to those of the duplex $(\mathrm{dG})_{18} \cdot(\mathrm{dC})_{18}$, which are $3.05 \AA$ and $30.8^{\circ}$, respectively. ${ }^{40} \mathrm{We}$ also remark that the quadruplex, despite the fact that it is composed of only three tetrads, shows reduced fluctuations $(\mathrm{rmsd}=0.18)$ of the helical rise compared to that of the much longer duplex (rmsd $=0.36$ ). Reduced fluctuations are also observed for the basepair opening and the buckle, which corresponds to contrarotation around the short axes of the base pair. The rmsd values found for buckle and base-pair opening in $\mathrm{d}(\mathrm{GGGG})_{4}$ are $7.9^{\circ}$ and $2.3^{\circ}$, respectively, against $9.8^{\circ}$ and $3.2^{\circ}$ for $(\mathrm{dG})_{18} \cdot(\mathrm{dC})_{18}$. The largest amplitude fluctuations in the quadruplex are related with the twist between the tetrads.

\subsection{Properties of the Franck-Condon Excited states}

By following the methodology described in Section 2.2 we determined the properties of the Franck-Condon excited states corresponding to each one of the 100 conformations of the quadruplex $\mathrm{d}(\mathrm{GGG})_{4}$. We can distinguish two ensembles of exciton states. Half of them are built on the $S_{1}$ state of the guanosine chromophore and the other half on the $S_{2}$ state, the former having lower energy than the latter.

Figure 6

Figure 6 shows the oscillator strength $f$ associated with each one of the 24 excited states averaged over 100 conformations. In contrast to the monomer for which the $S_{0} \rightarrow S_{1}$ and $S_{0} \rightarrow S_{2}$ transitions have quite similar oscillator strength, 0.19 and 0.21 , respectively, in the case of the quadruplex the $f$ values exhibit important dispersion. This picture contrasts with the ideal $\mathrm{H}$ or $\mathbf{J}$ aggregates, in which the oscillator strength is concentrated in the upper or the lower eigenstate, respectively. The existence of both in plane (within the tetrads) and out of plane (along the stacks) electronic couplings results in a more complex pattern. We 
remark in Figure 6 that eight excited states are characterized by $f$ values higher than 0.1 . For six among them $f$ is comprised between 0.01 and 0.1 , and the remaining appear to be completely “dark” $(f<0.01)$.

Figure 7

The distribution of the oscillator strength corresponding to the electronic transitions of 100 conformations, normalized per quadruplex, is presented in Figure 7, together with the two transitions of dGMP. In other terms, the plot in Figure 7 corresponds to the theoretical absorption spectrum of $\mathrm{d}(\mathrm{GGG})_{4}$. The total oscillator strength of the quadruplex transitions (4.6) is slightly lower that that of the constitutive monomers (4.8), indicating an overall hypochromism (4\%). The barycenter of the calculated quadruplex spectrum $\left(38940 \mathrm{~cm}^{-1}\right)$ is hypsochromically shifted by $350 \mathrm{~cm}^{-1}$ with respect to that of the monomer $\left(38590 \mathrm{~cm}^{-1}\right)$. The hypsochromic shift concerns both parts of the $3 \times \mathrm{G} 4$ spectrum, associated with the $S_{1}$ or the $S_{2}$ states of guanosine. For both ensembles, several very weak transitions are located at energies lower than that the corresponding transition of the monomeric chromophore. This is shown in the inset of Figure 7 focusing on the red side of the spectrum.

Figure 8

We quantified the degree of delocalization of the exciton states by the participation ratio $P R=1 / \mathrm{L}_{\mathrm{k}}$ which represents the number of coherently coupled chromophores: ${ }^{41,42}$

$$
\mathrm{L}_{\mathrm{k}}=\sum_{\text {monomer } m}\left[\sum_{\text {states } i}\left(C_{k, m}^{i}\right)^{2}\right]^{2}
$$

The sum within the square brackets represents the contribution to the eigenstate $\langle\mathrm{k}\rangle$ of 
different electronic states belonging to the same guanine. The average participation ratio, corresponding to each one of the 24 eigenstates of $d(\mathrm{GGG})_{4}$ is shown in Figure 8 . The PR values range from 5.4 (for $\langle\mathrm{k}\rangle=18$ ) to 11.1 (for $\langle\mathrm{k}\rangle=24$ ). In order to compare the degree of delocalization (D) of the quadruplex excited states with those determined for DNA duplexes according the same methodology, ${ }^{24,25,43}$ we divide the PR values by the number of bases composing the system. Thus, we find that all excited states of $\mathrm{d}(\mathrm{GGG})_{4}$ are delocalized over at least $45 \%$ of the guanines composing the quadruplex; the upper D value is as high as $95 \%$. The lower, upper and average $\mathrm{D}$ values determined for the quadruplex $\mathrm{d}(\mathrm{GGG})_{4}$ as well as for the double strands $(\mathrm{dG})_{10} \cdot(\mathrm{dC})_{10},(\mathrm{dA})_{10} \cdot(\mathrm{dT})_{10}$ and $(\mathrm{dAdT})_{5} \cdot(\mathrm{dAdT})_{5}$ are gathered on Table 3. It is clear that the excited states of the quadruplex are by far more delocalized than those of the duplexes despite the fact that the quadruplex is composed of much shorter strands (trimers) compared to the duplexes (decamers). This is understandable because, as explained in 4.1, the quadruplex structure undergoes weaker amplitude structural fluctuations leading to reduced off-diagonal disorder.

Table 3

Figure 9 shows the topography of two typical eigenstates of the quadruplex, $\langle 11>$ and $<1>$, both built on the $S_{1}$ state of guanosine. The former is associated with the strongest oscillator strength and it is located around $37500 \mathrm{~cm}^{-1}, e g$. close to the excitation conditions used in the experiments $(265 / 267 \mathrm{~nm})$. The latter is the lowest in energy eigenstate and is likely to be involved in emission. For the eigenstate $<11\rangle$ we observe a relatively uniform distribution of the coefficients $C_{i}$, which are comprised between 0.06 and 0.13 . In the case of the eigenstate $\left\langle 1>\right.$, the $C_{i}$ values show larger variations, ranging from 0.03 to 0.17 . 
Figure 9

\section{Discussion}

The experimental results presented in Section 3 revealed remarkable differences between the optical properties determined for G4-wires filled with $\mathrm{Na}^{+}$and the monomeric chromophore GMP. These differences concern both the shape of the absorption and fluorescence spectra and, in a more striking way, the fluorescence decays which are much slower in the case of the wires, the shortest time-constant $\tau_{1}$ being $c a .30 \mathrm{ps}$. Although longlived fluorescence components have been detected for various DNA duplexes under similar experimental conditions, the $\tau_{1}$ values do not exceed a few ps. ${ }^{19,44,45}$ The same is also true for the short quadruplex $\mathrm{d}(\mathrm{TGGGGT})_{4}$, for which $\tau_{1}$ is $c a .2 .6$ ps. ${ }^{11}$ The above observations reveal that arrangement of guanines in long four-stranded structures alters dramatically the properties of their electronic excited states. Our calculations provide useful insights in the specific behavior of the long G4-wires.

The molecular dynamics simulations showed that conformational motions, with the exception of twist, have relatively weak amplitude in $\mathrm{d}(\mathrm{GGG})_{4}$. Increasing the number of tetrads from 3 to $c a$. 800 is expected to further reduce structural disorder. Consequently, the collective behavior found for the Franck-Condon excited states in $\mathrm{d}(\mathrm{GGG})_{4}$, where edge effects are important, should more pronounced for the G4-wires.

In the calculation of the excited states we considered only dipolar coupling. However, orbital overlap interactions, which are difficult to compute for large multichromophoric systems, were shown to play an important role in short single and double DNA strands. ${ }^{46-51}$ Despite this limitation, the theoretical absorption spectrum in Figure 7 reproduces the main features of the experimental spectrum (Figure 3a). Both spectra are hypsochromically shifted with respect to that of the monomeric chromophore. The barycenter of the experimental 
spectrum of G4-wires is located $670 \mathrm{~cm}^{-1}$ at higher energy compared to that of dGMP. The same trend but smaller in amplitude $\left(350 \mathrm{~cm}^{-1}\right)$ is found for the spectrum calculated for $\mathrm{d}(\mathrm{GGG})_{4}$. We note that a size dependent blue shift was reported for the experimental absorption spectra of $(\mathrm{dA})_{\mathrm{n}} \cdot(\mathrm{dT})_{\mathrm{n}}$, the largest value corresponding to the longest duplex. ${ }^{21}$ Moreover, the seven eigenstates of $\mathrm{d}(\mathrm{GGG})_{4}$ lying at lower energy than the $\mathrm{S}_{1}$ state of guanosine (inset in Figure 7) could account for the red tail of the experimental spectrum. According to the calculations, these low lying states are devoid of oscillator strength. However, in a real system, due to vibrational coupling, they may borrow oscillator strength from the close lying bright states. This mechanism should result in a decrease of the shoulder corresponding to $S_{0} \rightarrow S_{1}$ transition and in the appearance of the red tail observed in the experimental spectra (Figure 3a).

The calculations concern only the Franck-Condon exciton states, populated directly by photon absorption. As a general rule, two competing processes may take place before emission: intraband scattering and localization of the excited states. Intraband scattering, eg. internal conversion among exciton states, brings very rapidly the system to the bottom of the exciton band. This process was shown to occur on the femtosecond time-scale for DNA duplexes ${ }^{20-22,44}$ as well as for the four stranded d(TGGGGT). ${ }^{11}$ Emission may originate from low lying states corresponding to weakly allowed transitions. However vibrational and conformational motions tend to reduce the spatial extent of the excited states. Consequently, emission may originate from excited states less delocalized than the Franck-Condon ones, even from monomeric chromophores. It is also possible that an excited monomeric chromophore gives rise to an excimer corresponding to the minimum of the potential energy surface of an excited dimer.

The fluorescence decays of dGMP follow complex kinetics ${ }^{38}$ attributed to emission from different regions of the potential energy surface of the $S_{1}$ state $\left(\pi \pi^{*}\right) .{ }^{52}$ But the excited 
state relaxation is ultrafast, the average lifetime not exceeding $1 \mathrm{ps}^{38}{ }^{38}$ It would not be surprising if some non-radiative deactivation pathways leading to the ground state through a conical intersection $^{52}$ were blocked within the quadruplex, as it happens in low temperature glasses $^{13}$ resulting to a lengthening of the fluorescence lifetime. As a matter of fact, the intrachromophore structural changes responsible for the ultrafast excited state deactivation could be hindered by four Hoogsteen hydrogen bonds linking each guanosine to the others (Figure 1) and the stacking of tetrads. In such a case, emission would arise from the FranckCondon region. This interpretation is not corroborated by the position of the fluorescence maximum of the wires which is red-shifted by $2176 \mathrm{~cm}^{-1}$ with respect to that of the dGMP. Moreover, if emission originated from excited states localized on individual guanines, the radiative lifetime should be close to that observed dGMP (4 ns). Though we cannot rule out the possibility that the shortest time constant is related to monomer emission, the data on Table 2 indicate that the largest part of the emitted photons is related to quasi dark states. Therefore, we conclude that emission from $\pi \pi^{*}$ states localized on individual guanines is not the dominant process.

Excimer emission is characterized by a broad band peaking at lower energy compared to the emission of the monomeric chromophore. Such a fluorescence, peaking at $430 \mathrm{~nm}$ was detected for the d(TGGGGT) single strand but disappears upon formation of the quadruplex. ${ }^{11}$ Although the fluorescence spectrum of the G4-wires peaks at lower energies compared to that of dGMP, its barycenter is located at higher energy; its shape is more symmetrical and its width is smaller. All these features do not support the hypothesis of excimer emission.

Following the above reasoning, we conclude that the dominant emitting species in the G4-wires are exciton states. These may be populated either directly from intraband scattering or result from further relaxation due to restricted conformational motions during the lifetime of the excited state. It is worth-noticing that about half of the emitted photons are associated 
with a time constant of $2 \mathrm{~ns}$. We are tempted to assign this emission to individual tetrads which could behave as a single chromophore. This is supported by the observation that the largest amplitude motion found for $\mathrm{d}(\mathrm{GGG})_{4}$ corresponds to the twist between the tetrads. In such a scenario, G4-wires would behave a columnar phases in which, after initial ultrafast scattering, excitation energy may be transferred along the column axis via a hopping mechanism..$^{53,54}$

\section{Remarks and Perspectives}

The work presented here highlighted the collective behavior of the excited states of G4wires. Not only the Franck-Condon excited states but also the emitting states are delocalized due to restricted conformational motions specific of the quadruplex structure and enhanced by the size of the wires. Several questions arise from this first combined experimental and theoretical study on the electronic excited states of G-quadruplexes.

The first question concerns the role metal cations. The fluorescence decays reported here for G4-wires filled with $\mathrm{Na}^{+}$are longer $\left(\tau_{1} \approx 30 \mathrm{ps}\right)$ that those obtained for a similar system either empty or filled with $\mathrm{K}^{+}\left(\tau_{1}<10 \mathrm{ps}\right) .{ }^{13}$ Systematic measurements with various metal cations are necessary in order to elucidate this point. Quantum chemical calculations could provide an insight about the way (structural, electronic) that the metal cations interfere with the excited states. Furthermore, the influence of the quadruplex size on the optical properties needs to be examined. We mentioned that the number of tetrads is expected to reduce conformational disorder and enhance the collective behavior of the excited states. However, one could imagine the existence of a coherence length. A related question is whether the electronic coherence length, determined by delocalization of the excited states along the axis of the wires, coincides with the structural coherence length. Finally, it will be interesting to characterize the various regimes of excitation transport (intraband scattering, excitation hopping) and compare them with processes occurring in columnar liquid crystals formed by 
discotic molecules. ${ }^{54}$ Answering the above questions will contribute to design guanine wires with improved optical properties which could possibly be integrated in molecular electronic devices.

Acknowledgment: This work was supported by NanoSciences Ile-de-France (project $\mathrm{N}^{\circ}$ IF-06-357/R-9), Laser Lab Europe and the COST Action MP0802 (G4-NET). We thank Dr Richard Lavery for helpful discussions. 


\section{References}

(1) Yan, H.; Zhang, X. P.; Shen, Z. Y.; Seeman, N. C. Nature 2002, 415, 62.

(2) Kwon, Y. W.; Lee, C. H.; Choi, D. H.; Jin, J. I. J. Mat. Chem. 2009, 19, 1353.

(3) Kotlyar, A. B.; Borovok, N.; Molotsky, T.; Cohen, H.; Shapir, E.; Porath, D. Adv. Mater. 2005, 17, 1901.

(4) Shapir, E.; Sagiv, L.; Borovok, N.; Molotski, T.; Kotlyar, A. B.; Porath, D. J. Phys. Chem. B 2008, 112, 9267.

(5) De Cian, A.; Lacroix, L.; Douarre, C.; Temime-Smaali, N.; Trentesaux, C.; Riou, J. F.; Mergny, J. L. Biochimie 2008, 90, 131.

(6) Brooks, T. A.; Hurley, L. H. Nature Reviews Cancer 2009, 9, 849.

(7) Franceschin, M. Eur. J. Org. Chem. 2009, 2225.

(8) Luedtke, N. W. Chimia 2009, 63, 134.

(9) Wong, H. M.; Payet, L.; Huppert, J. L. Curr. Opin. Mol. Therap. 2009, 11, 146.

(10) Mergny, J. L.; De Cian, A.; Ghelab, A.; Sacca, B.; Lacroix, L. Nucleic Acids Res. $2005,33,81$.

(11) Miannay, F. A.; Banyasz, A.; Gustavsson, T.; Markovitsi, D. J. Phys. Chem. C 2009, 113, 11760.

(12) Markovitsi, D.; Gustavsson, T.; Sharonov, A. Photochem. Photobiol. 2004, 79, 526.

(13) Gepshtein, R.; Huppert, D.; Lubitz, I.; Amdursky, N.; Kotlyar, A. B. J. Phys. Chem. C 2008, 112, 12249 .

(14) Mendez, M. A.; Szalai, V. A. Biopolymers 2009, 91, 841.

(15) Schwalb, N. K.; Temps, F. Science 2008, 322, 243.

(16) McGovern, D. A.; Quinn, S.; Doorley, G. W.; Whelan, A. M.; Ronayne, K. L.; Towrie, M.; Parker, A. W.; Kelly, J. M. Chem. Comm. 2007, 5158.

(17) Hud, V.; Plavec, J. The role of cations in determining quadruplex structure and stability. In Quadruplex nucleic acids; Balasubramanian, S., Neidle, S., Eds.; RSC Publishing: Cambridge, 2006; pp 100.

(18) Markovitsi, D.; Onidas, D.; Gustavsson, T.; Talbot, F.; Lazzarotto, E. J. Am. Chem. Soc. 2005, 127, 17130.

(19) Markovitsi, D.; Talbot, F.; Gustavsson, T.; Onidas, D.; Lazzarotto, E.; Marguet, S. Nature 2006, 441, E7.

(20) Onidas, D.; Gustavsson, T.; Lazzarotto, E.; Markovitsi, D. Phys. Chem. Chem. Phys. 2007, 9, 5143 .

(21) Onidas, D.; Gustavsson, T.; Lazzarotto, E.; Markovitsi, D. J. Phys. Chem. B 2007, 111, 9644 .

(22) Miannay, F. A.; Banyasz, A.; Gustavsson, T.; Markovitsi, D. J. Am. Chem. Soc. 2007, 129, 14574.

(23) Markovitsi, D.; Onidas, D.; Talbot, F.; Marguet, S.; Gustavsson, T.; Lazzarotto, E. J. Photochem. Photobiol. A: Chem. 2006, 183, 1.

(24) Bouvier, B.; Dognon, J. P.; Lavery, R.; Markovitsi, D.; Millié, P.; Onidas, D.; Zakrzewska, K. J. Phys. Chem. B 2003, 107, 13512.

(25) Emanuele, E.; Zakrzewska, K.; Markovitsi, D.; Lavery, R.; Millie, P. J. Phys. Chem. B 2005, 109, 16109.

(26) Rashbah, E. I.; Sturge, M. D. Excitons; North-Holland: Amsterdam, 1982. 
(27) Shapir, E.; Sagiv, L.; Borovok, N.; Molotski, T.; Kotlyar, A. B.; Porath, D. Journal of Physical Chemistry B 2008, 112, 9267.

(28) Borovok, N.; Molotsky, T.; Ghabboun, J.; Porath, D.; Kotlyar, A. Anal. Biochem. 2008, 374, 71.

(29) Onidas, D.; Markovitsi, D.; Marguet, S.; Sharonov, A.; Gustavsson, T. J. Phys. Chem. B 2002, 106, 11367 .

(30) Berendsen, H. J. C.; Postma, J. P. M.; van Gunsteren, W. F.; DiNola, A.; Haak, J. R. J. Chem. Phys. 1984, 81, 3684.

(31) Ryckaert, J. P.; Ciccotii, G.; Berendsen, H. J. C. J. Comp. Phys. 1977, 23, 327.

(32) Darden, T.; York, D.; Pedersen, L. J. Chem. Phys. 1993, 98, 10089.

(33) Sharp, K. A.; Honig, B. Annu. Rev. Biophys. Biophys. Chem. 1990, 19, 301.

(34) Bouvier, B.; Gustavsson, T.; Markovitsi, D.; Millié, P. Chem. Phys. 2002, 275, 75 .

(35) Orozco, M.; Noy, A.; Perez, A. Curr. Opin. Struct. Biol. 2008, 18, 185.

(36) Claverie, P. Persp. Quant. Chem. Biochem. 1978, 2(Intermolecular Interactions - From Diatomics to Biopolymers), 69.

(37) Pople, J. A.; Beveridge, D. L.; Dobosh, P. A. J. Chem. Phys. 1967, 47, 2026.

(38) Miannay, F. A.; Gustavsson, T.; Banyasz, A.; Markovitsi, D. J. Phys. Chem. A 2010, 114, 3256 .

(39) Phillips, K.; Dauter, Z.; Murchie, A. I. H.; Lilley, D. M. J.; Luisi, B. J. Mol. Biol. 1997, 273, 171.

(40) Lavery, R.; Zakrzewska, K.; Beveridge, D.; Bishop, T. C.; Case, D. A.; Cheatham, T.; Dixit, S.; Jayaram, B.; Lankas, F.; Laughton, C.; Maddocks, J. H.; Michon, A.; Osman, R.; Orozco, M.; Perez, A.; Singh, T.; Spackova, N.; Sponer, J. Nucleic Acids Res., 38, 299.

(41) Dean, P. Rev. Mod. Phys. 1972, 44, 127.

(42) Schreiber, M.; Toyosawa, Y. J. Phys. Soc. Jpn. 1982, 51, 1537.

(43) Markovitsi, D.; Gustavsson, T.; Banyasz, A. DNA Fluorescence. In CRC Handbook of Organic Photochemistry and Photobiology Griesbeck, A., Ghetti, F., Oelgemoeller, M., Eds.; Taylor and Francis: Boca Raton, 2010; pp in press.

(44) Markovitsi, D.; Gustavsson, T.; Talbot, F. Photochem. \& Photobiol. Sci. 2007, $6,717$.

(45) Vayá, I.; Miannay, F. A.; Gustavsson, T.; Markovitsi, D. ChemPhysChem 2010, $11,987$.

(46) Starikov, E. B. Modern Phys. Lett. B 2004, 18, 825.

(47) Starikov, E. B.; Cuniberti, G.; Tanaka, S. J. Phys. Chem. B 2009, 113, 10428.

(48) Santoro, F.; Barone, V.; Improta, R. Proc. Natl. Acad. Sci. 2007, 104, 9931.

(49) Santoro, F.; Barone, V.; Improta, R. ChemPhysChem 2008, 9, 2531.

(50) Santoro, F.; Barone, V.; Importa, R. J. Am. Chem. Soc. 2009, 131, 15232.

(51) Lange, A. W.; Herbert, J. M. J. Am. Chem. Soc. 2009, 131, 3913.

(52) Karunakaran, V.; Kleinermanns, K.; Improta, R.; Kovalenko, S. A. J. Am. Chem. Soc. 2009, 131, 5839 .

(53) Markovitsi, D.; Germain, A.; Millie, P.; Lécuyer, I.; Gallos, L.; Argyrakis, P.; Bengs, H.; Ringsdorf, H. J. Phys. Chem. 1995, 99, 1005.

(54) Markovitsi, D. Mol. Cryst. Liq. Cryst. 2003, 397, 389. 
10/09/2010 
TABLE 1: Comparison of the optical properties determined experimentally for G4wires and dGMP

\begin{tabular}{|c|c|c|c|c|}
\hline & property & dGMP & G4-wires & wire effect \\
\hline \multirow{4}{*}{$\begin{array}{c}\text { absorption } \\
\text { spectra }\end{array}$} & maximum $\left(\mathrm{cm}^{-1}\right)$ & 39526 & 39841 & $\Uparrow 315$ \\
\cline { 2 - 5 } & barycenter $\left(\mathrm{cm}^{-1}\right)$ & 39100 & 39670 & $\Uparrow 570$ \\
\cline { 2 - 5 } & width $\left(\mathrm{cm}^{-1} ;\right.$ fwhm $)$ & 7100 & 6600 & $\Downarrow 500$ \\
\cline { 2 - 5 } & red tail $\left(\mathrm{I}_{295 \mathrm{~nm}} / \mathrm{I}_{\mathrm{max}}\right)$ & $6 \%$ & $17 \%$ & $\Uparrow$ factor 2.8 \\
\cline { 2 - 5 } emission & maximum $\left(\mathrm{cm}^{-1}\right)$ & 29500 & 27300 & $\Downarrow 2200$ \\
\cline { 2 - 5 } spectra & barycenter $\left(\mathrm{cm}^{-1}\right)$ & 23900 & 25500 & $\Uparrow 1600$ \\
\cline { 2 - 5 } & width $\left(\mathrm{cm}^{-1} ; \mathrm{fwhm}\right)$ & 8,950 & 8,300 & $\Downarrow 650$ \\
\cline { 2 - 5 } & red tail $\left(\mathrm{I}_{555 \mathrm{~nm}} / \mathrm{I}_{\mathrm{max}}\right)$ & $29 \%$ & $8 \%$ & $\Downarrow$ factor 3.6 \\
\cline { 2 - 5 } & quantum yield & $(1.3 \pm 0.1) \times 10^{-4}$ & $(9.5 \pm 0.1) \times 10^{-4}$ & $\Uparrow$ factor 7.3 \\
\hline \multirow{2}{*}{$\begin{array}{c}\text { average } \\
\text { lifetimes }\end{array}$} & fluorescence $(\mathrm{ps})$ & 0.52 & 136 & $\Uparrow$ factor 261 \\
\cline { 2 - 5 } & radiative $(\mathrm{ns})$ & 4 & 146 & $\Uparrow$ factor 36 \\
\hline
\end{tabular}


TABLE 2: Parameters derived from the fits of the fluorescence decays of G4-wires with tri-exponential functions $\Sigma \mathbf{a}_{\mathbf{i}} \exp \left(-\mathrm{t} / \tau_{\mathrm{i}}\right) ; \mathbf{p}_{\mathbf{j}}=\mathbf{a}_{\mathbf{j}} \tau_{\mathbf{j}} /\left(\Sigma \mathbf{a}_{\mathrm{i}} \tau_{\mathrm{i}}\right)$ is the weight corresponding to the time-constant $\tau_{\mathbf{j}}$ (in ps) and $\langle\tau\rangle=\Sigma \mathrm{a}_{\mathrm{i}} \tau$ represents the average lifetime.

\begin{tabular}{ccc} 
& $\mathbf{3 4 0} \mathbf{~ n m}$ & $\mathbf{4 5 0} \mathbf{~ n m}$ \\
\hline $\mathbf{a}_{\mathbf{1}}$ & $0.808 \pm 0.020$ & $0.698 \pm 0.002$ \\
$\boldsymbol{\tau}_{1}$ & $31.6 \pm 0.2$ & $25.8 \pm 0.4$ \\
$\mathbf{p}_{\mathbf{1}}$ & 0.194 & 0.109 \\
$\mathbf{a}_{\mathbf{2}}$ & $0.161 \pm 0.004$ & $0.269 \pm 0.001$ \\
$\boldsymbol{\tau}_{2}$ & $274 \pm 2$ & $302 \pm 2$ \\
$\mathbf{p}_{\mathbf{2}}$ & 0.335 & 0.492 \\
$\mathbf{a}_{3}$ & $0.031 \pm 0.001$ & $0.0330 \pm 0.0002$ \\
$\boldsymbol{\tau}_{3}$ & $2000 \pm 20$ & $2000 \pm 40$ \\
$\mathbf{p}_{\mathbf{3}}$ & 0.471 & 0.399 \\
$\langle\tau>$ wires & 132 & 165 \\
$\langle\tau>$ dGMP & 0.42 & 0.94
\end{tabular}


TABLE 3: Degree of delocalization (D) determined for the Franck-Condon excited states of DNA double and quadruple strands. It is obtained by dividing the participation ratio (conformation average) by the number of bases composing the system.

\begin{tabular}{ccccc} 
system & $\mathbf{D}_{\mathbf{m i n}}$ & $\mathbf{D}_{\mathbf{m a x}}$ & $\langle\mathbf{D}\rangle^{\mathbf{a}}$ & Ref. \\
\hline $\mathbf{d}(\mathbf{G G G})_{\mathbf{4}}$ & 0.45 & 0.95 & 057 & This work \\
$(\mathbf{d G})_{\mathbf{1 0}} \cdot(\mathbf{d C})_{\mathbf{1 0}}$ & 0.21 & 0.59 & 0.28 & 25,43 \\
$(\mathbf{d A})_{\mathbf{1 0}} \cdot(\mathbf{d T})_{\mathbf{1 0}}$ & 0.20 & 0.36 & 0.29 & 24 \\
$(\mathbf{d A d T})_{\mathbf{5}} \cdot(\mathbf{d A d T})_{\mathbf{5}}$ & 0.17 & 0.42 & 0.27 & 24
\end{tabular}

a) double average, first over the conformations and then over the various excited states of the system. 


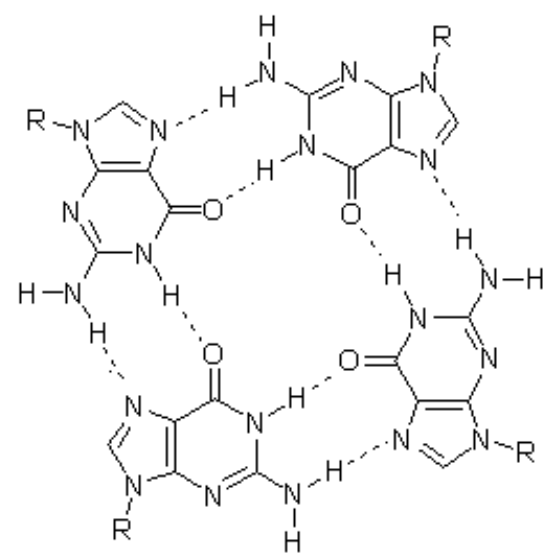

Figure 1. Schematic representation of the guanine tetrad formed via Hoogsteen hydrogen bonding. 


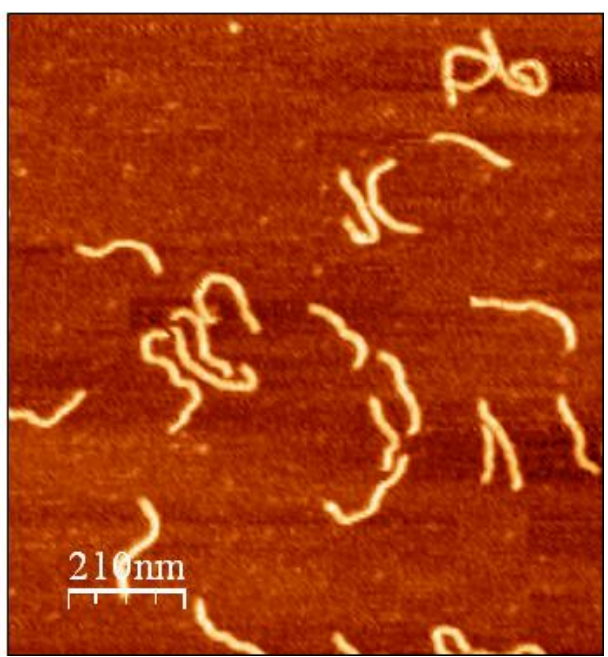

Figure 2. AFM image of the G4-wires used in the study 


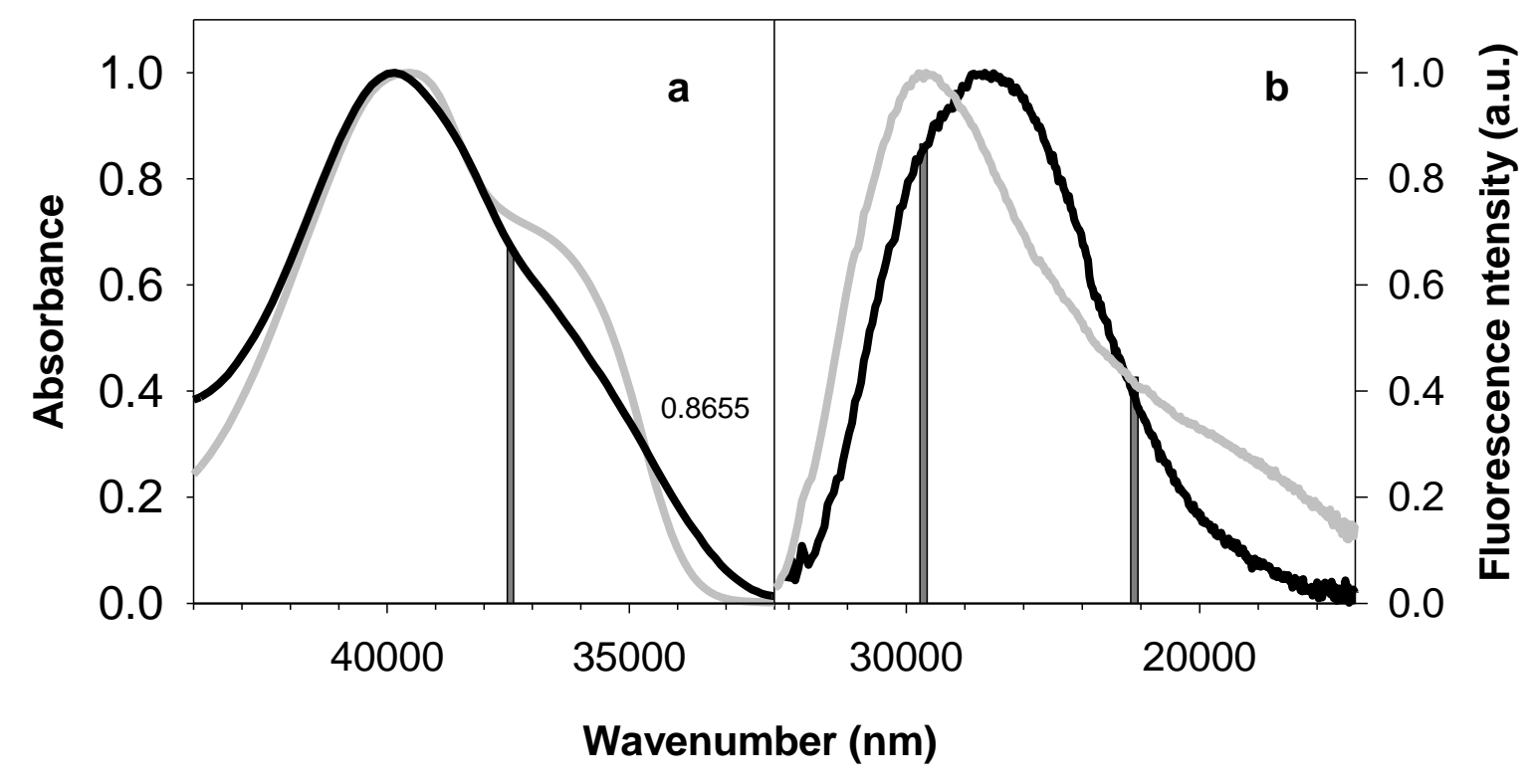

Figure 3: Normalized absorption (a) and fluorescence (b) spectra of G4-wires (black) and dGMP (grey; concentration $10^{-5} \mathrm{M}$ ). Vertical bars correspond to the excitation wavelength $(265 \mathrm{~nm})$ and to the emission wavelengths at which the fluorescence decays were recorded (340 and $450 \mathrm{~nm}$ ). In order to convert the fluorescence spectra from wavelength $(\lambda)$ to wavenumber scale, their intensity I at a given $\lambda$ was multiplied by $\mathrm{I} \lambda^{2}$. 


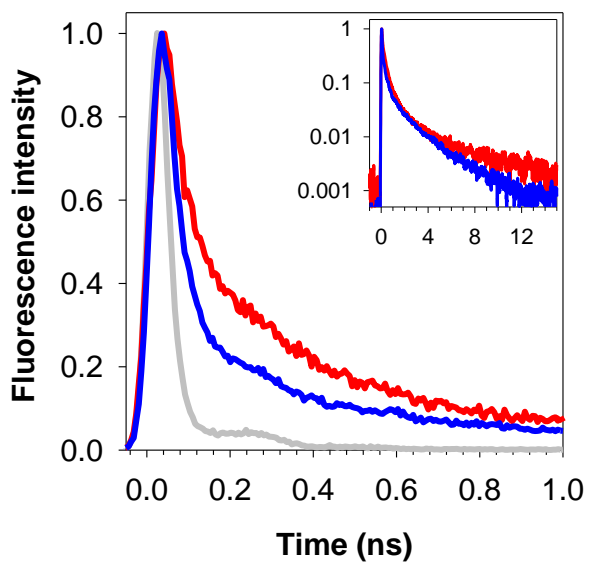

Figure 4. Fluorescence decays of G4-wires recorded at $340 \mathrm{~nm}$ (blue) and $450 \mathrm{~nm}$ (red) following excitation at $267 \mathrm{~nm}$. The apparatus response function is shown in grey. 

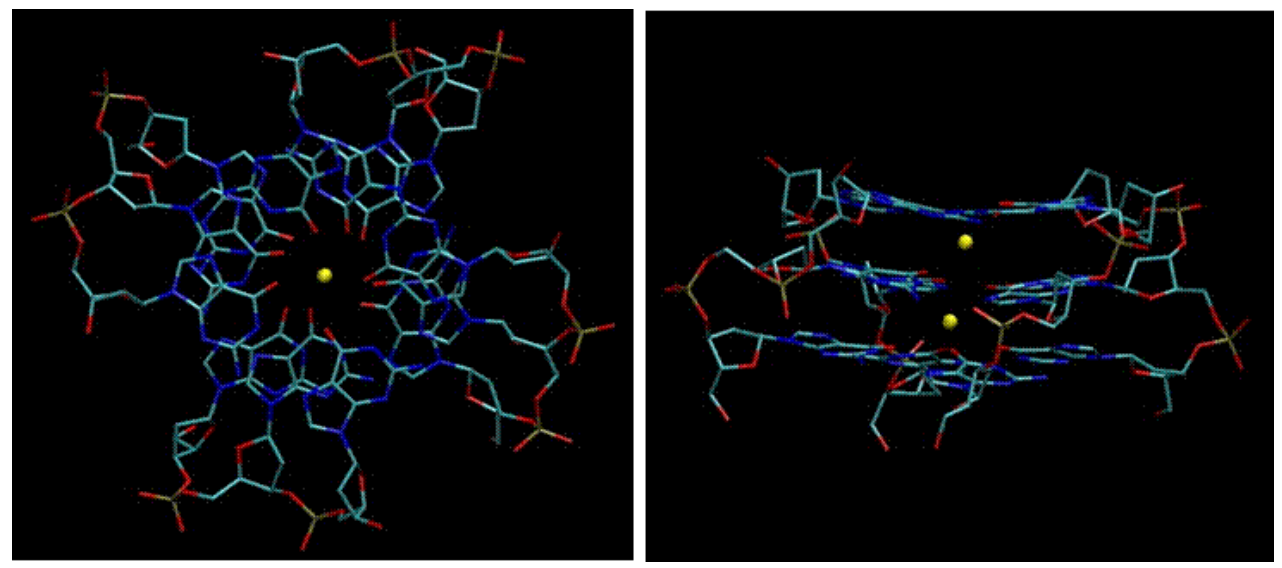

Figure 5. Top and side views of the $\mathrm{d}(\mathrm{GGG})_{4}$ quadruplex structure derived from molecular dynamics calculations. Sodium cations (yellow) are located in the central cavity. 


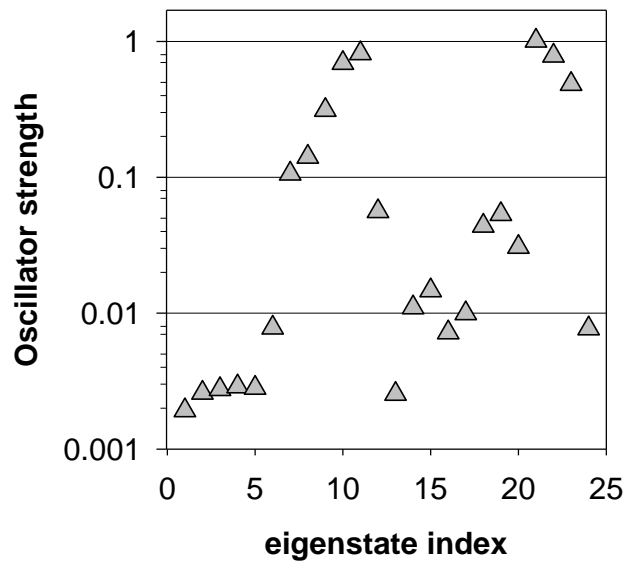

Figure 6. Oscillator strength associated with each one the 24 eigenstates of the $d(G G G)_{4}$ quadruplex. Average values for 100 conformations. 


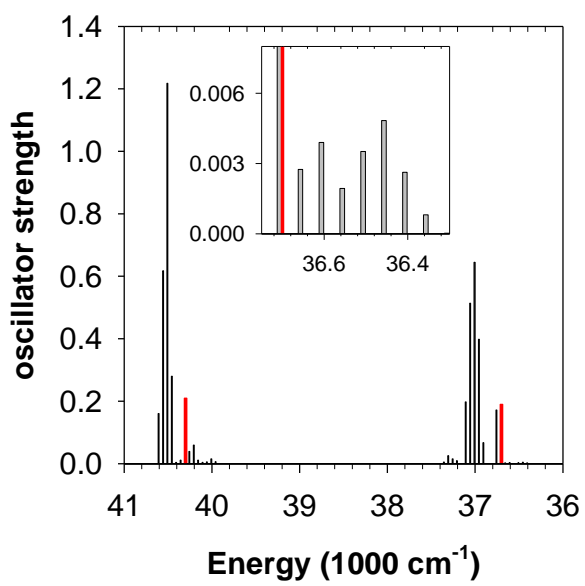

Figure 7. Distribution of the oscillator strength calculated for 100 conformations of the $\mathrm{d}(\mathrm{GGG})_{4}$ quadruplex. The total oscillator strength is normalized per quadruplex; the width of the individual subdivisions is equal to $10 \mathrm{~cm}^{-1}$. The $S_{1}$ and the $S_{2}$ states of dGMP denoted by red bars. Inset: low lying eigenstates associated with weak oscillator strength. 


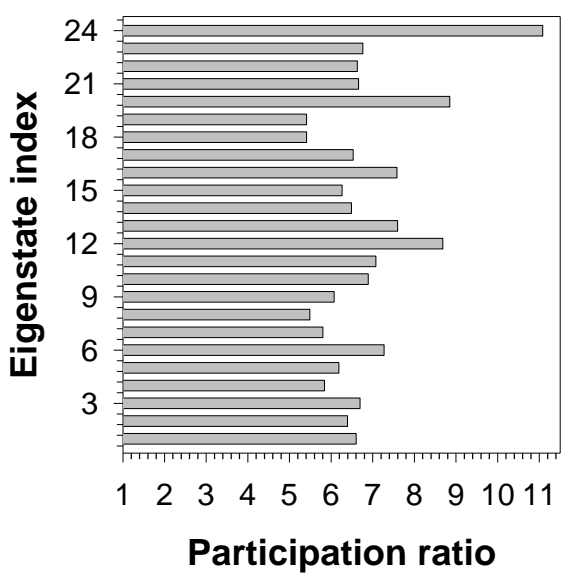

Figure 8. Participation ratio representing the number of coherently coupled bases for each eigenstate of the $\mathrm{d}(\mathrm{GGG})_{4}$ quadruplex. Average values for 100 conformations. 


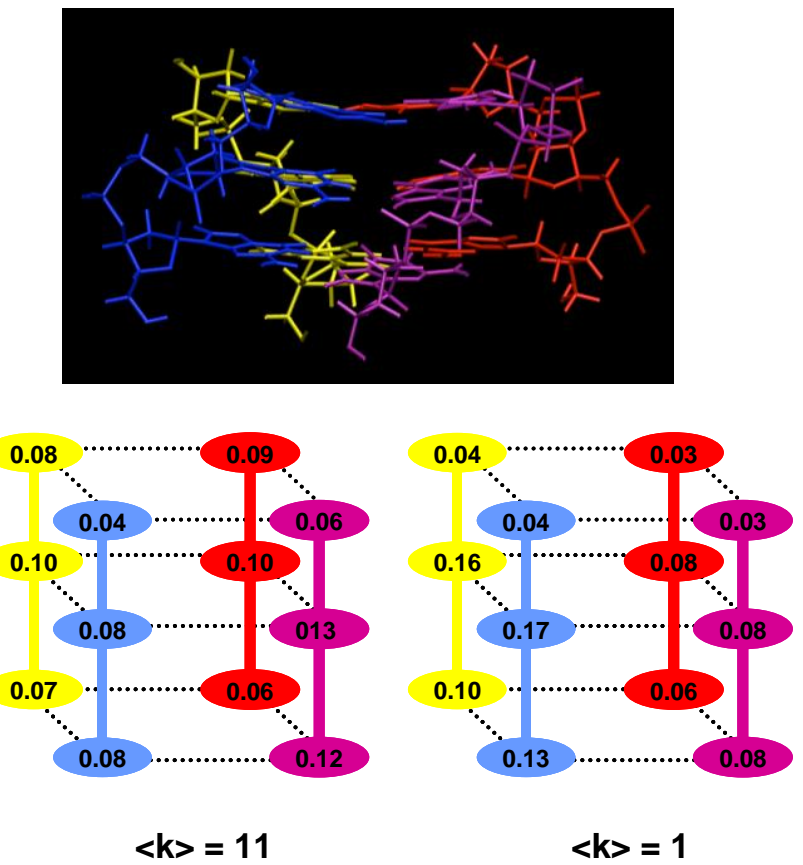

Figure 9. Topographies of two typical excited states calculated for the quadruplex d(GGG) built on the $S_{1}$ state of the guanosine chromophore. Each tri-nucleotide is represented by a specific color. The numbers correspond to the contribution of each base to the exciton state. 